\title{
NAS TRILHAS DE LEO WAIBEL: PESQUISAS ALEMÃS DE GEOGRAFIA HUMANA DO BRASIL - DE HEIDELBERg A TÜBINGEN $(1950-2005)^{*}$
}

\author{
Gerd Kohlhepp \\ Universität Tübingen \\ gerd.kohlhepp@t-online.de
}

Resumo

A cooperação entre a geografia alemã e brasileira, aspirada por Waibel, foi iniciada em 1950 por seu aluno Pfeifer que ampliou consideravelmente a temática das pesquisas da geografia econômica e regional sobre o Brasil na Universidade de Heidelberg. Kohlhepp, colaborador de Pfeifer iniciou então os estudos sobre geografia industrial e agrária no sul do Brasil. A partir de 1972, Kohlhepp atuava em Frankfurt fundando no Instituto de Geografia em Tübingen, a partir de 1978, o Centro de Pesquisas sobre a América Latina (CPAL) com enfoque no Brasil e com estreita cooperação bilateral. Os projetos de pesquisa na Amazônia, no Brasil Central, no Sudeste e Sul trataram de temas como a colonização agrária, frentes pioneiras e desenvolvimento regional, mudança da estrutura agrária, globalização, regionalização, megaprojetos, desenvolvimento urbano nas cidades pioneiras e metrópoles como também problemas de meio ambiente. Kohlhepp como professor emérito em 2005 e Coy atendendo convite para atuar em Innsbruck (Austria), as pesquisas da geografia humana sobre o Brasil terminaram em Tübingen, sendo hoje levadas adiante por outros Institutos.

Palavras-chave: Pesquisas sobre Brasil; geografia humana alemã; Waibel; Pfeifer; Kohlhepp; Coy; CPALTübingen.

\begin{abstract}
Cooperation between Brazilian and German geography desired by Waibel during his stay in Brazil started in 1950 at the University of Heidelberg with Pfeifer who strongly broadened the range of topics in research on Brazil in human and regional geography. Studies in industrial and agrarian geography were elaborated by Pfeifer's assistant Kohlhepp who was offered a chair at Frankfurt in 1972 and at Tübingen in 1978 where he installed a Centre of Latin American Research with Brazil as main area and a strong bilateral cooperation. Research projects in Amazonia, Central, Southeast and South Brazil were focused on agricultural colonization, pioneer front and regional development, changes of agrarian structure, globalization, regionalization, megaprojects, urban development in pioneer towns and metropolitan areas and environmental problems. When Kohlhepp became professor emeritus in 2005 and Coy was appointed professor at Innsbruck/Austria research on Brazil came to an end in Tübingen and nowadays is continued by other Institutes in Germany.
\end{abstract}

Key words: Research on Brazil; german human geography; Waibel; Pfeifer; Kohlhepp; Coy; CPAL-Tübingen

eo Waibel proferiu seu famoso discurso de despedida em 17 de agosto de 1950 no ConseIho Nacional de Geografia no Rio de Janeiro, onde havia ocupado o cargo de consultor científico desta Instituição estando assim a serviço do Governo Brasileiro. Devido à expulsão de sua cátedra na Universidade de Bonn ${ }^{1}$ por motivos políticos, Waibel teve que emigrar ${ }^{2}$ para

\footnotetext{
* Esta contribuição trata de uma visão histórica (1950-2005) sobre a geografia humana alemã na pesquisa sobre o Brasil - exclusivamente nas Universidades de Heidelberg, Frankfurt e especialmente em Tübingen.

${ }^{1}$ Waibel, cuja esposa era judia, apesar do seu renome internacional, foi suspendido de todas as suas funções e atividades em universidades alemãs devido às leis raciais dos tempos nazistas. Sua posição contrária ao regime era conheci-
} 
os Estados Unidos em 1939 obtendo a cidadania norte americana naquele exílio em 1945. Isto facilitou sua contratação pelo CNG em 1946. Para a chefia desta instituição os intensos trabalhos científicos de Waibel junto com jovens geógrafos brasileiros, extremamente talentosos que Waibel chamava de "terceira geração de alunos" depois do tempo na Alemanha e nos EUA, foram considerados um grande sucesso. ${ }^{3}$ Os efeitos posteriores positivos do trabalho de Waibel - personalidade de pesquisador como mestre a nivel teórico e nos estudos de campo - mostram os cargos de liderança que uma série de seus colaboradores conseguiram no CNG e em outras instituições. Para citar alguns - Fábio de Macedo Soares Guimarães, Orlando Valverde, Lysia e Nilo Bernardes, Miguel Alves de Lima ou Walter Egler como também Speridião Faissol e Pedro P. Geiger como colaboradores temporários.

Em seu discurso "O que aprendi no Brasil" (WAIBEL, 1950), Waibel deixou claro que conhecimentos importantes resultaram para ele como geógrafo no Brasil e enriqueceram decisivamente os fundamentos da planejada "geografia dos trópicos". Ele acentuou que “... é necessária a pesquisa de campo e a concepção teórica para, do conjunto dos fatos isolados podermos tirar um princípio ordenador" (1950, p. 428). Perante o desafio da enorme área do país ele não se concentrou à geografia regional, mas a dois problemas da geografia sistemática: a utilização da terra e a colonização agrária. Quanto à solução de problemas de desenvolvimento do país e, numa fase na qual a geografia ainda tinha que se posicionar como ciência complexa, valia para ele, "[...] nenhuma ciência é mais indicada de que a geografia" (p. 428). Ele entrosava seus colaboradores também nos métodos da pesquisa geográfica alemã, uma vez que no Brasil os trabalhos dos colegas franceses e norte-americanos eram, até então, mais conhecidos.

Em seus trabalhos, Waibel achava importante incluir, além da literatura geográfica, estudos de historiadores e sociólogos brasileiros. ${ }^{4}$ As publicações mais importantes de Waibel no Brasil como os estudos em Goiás, a vegetação e o uso da terra no Planalto Central, os princípios da colonização européia no sul do Brasil, as zonas pioneiras do Brasil e o discurso de despedida foram publicadas na Revista Brasileira de Geografia. ${ }^{5}$

Durante suas atividades no Brasil foi importante para Waibel (1888-1951) convidar seu antigo aluno e colaborador Gottfried Pfeifer (1901-85) com o qual mantinha estreito relacionamento científico. Devido a dificuldades burocráticas e organisacionais da Alemanha pós-guerra, o encontro somente pôde ser realizado em maio de 1950, pouco antes do término do seu contrato com o CNG. Assim, Pfeifer teve a sorte de ser um dos primeiros geógrafos alemães que puderam pesquisar ultramar depois da segunda guerra mundial. Waibel queria despertar o interesse de Pfeifer pela geografia dos trópicos e estava convencido de que a terminologia da geografia humana, de-

\footnotetext{
da e ele negou divorciar-se de sua esposa, alternativa sugerida pela administração política. Vide KOHLHEPP, 2013 e correspondência privada de Waibel e seu aluno G. Pfeifer (arquivo particular do autor em Tübingen).

2 Waibel atuava nos Estados Unidos como Research Associate junto a I. Browman, de 1939-41. De 1941-44 ele pôde substituir um colega como professor em Madison/Wisc. Nos anos de 1944-46 ele foi colaborador do "Project M" do governo dos Estados Unidos, que estudava as possibilidades de assentamento de refugiados europeus na América Central depois do final da segunda guerra mundial (BELL, 2016; KOHLHEPP, 2013).

${ }^{3}$ VALVERDE $(1968)$, BERNARDES $(1952,1983)$ e informações pessoais dos citados antigos colaboradores de Waibel. A atividade de Waibel no Brasil mereceu inúmeras homenagens póstumas em necrológios na Alemanha e nos Estados Unidos.

${ }^{4}$ Assim p.ex. Capistrano de Abreu, Oliveira Viana, Caio Prado Jr. ou Gilberto Freyre.

${ }^{5}$ Estas publicações foram traduzidas para o alemão por G. Kohlhepp (PFEIFER \& KOHLHEPP, 1984), o discurso de despedida também em PFEIFER, 1971. Os trabalhos sobre o Planalto Central e Sul do Brasil também foram publicados como artigos no Geographical Review $(1948,1950)$.
} 
senvolvida nas zonas temperadas não era adequada para os trópicos. Ele defendia a idéia de uma disciplina que deveria chamar-se "geografia tropical". Além disso, Waibel queria realizar algumas das grandes excursões oficiais com Pfeifer, anteriormente planejadas que o CNG generosamente também financiou para Pfeifer. Com Pfeifer, a geografia alemã conseguiu, pela primeira vez, um novo acesso direto ao Brasil. Pelo falecimento precoce de Waibel em 1951, durante a sua primeira viagem à Alemanha depois da sua expulsão de Bonn, trabalhos conjuntos e anteriormente planejados não puderam mais ser realizados.

Para os adeptos da geografia no Brasil, o caminho científico de Pfeifer antes do início dos seus trabalhos no Brasil devem ser brevemente mencionados: depois da sua tese de doutorado junto à Waibel na Universidade de Kiel em 1927 sobre a geografia de povoamento de uma região na Alemanha do Norte, tese esta que documentava sua estreita ligação com a história em secções transversais de períodos históricos, dedicou-se aos critérios da ordem espacial da economia à luz de estudos científicos de economistas. ${ }^{6}$ Nisso ele também se apoiou em modelos de análise do espaço econômico que levou Waibel ao conceito da "formação econômica"7 em seus trabalhos na Sierra Madre de Chiapas no México em 1927. A contribuição de Pfeifer levou ao desenvolvimento da geografia econômica moderna para a pesquisa de estruturas espaciais.

Durante uma estada de quatro anos junto à Universidade da Califórnia em Berkeley (192832) onde trabalhou com Carl $O$. Sauer, pesquisador de carismática personalidade que marcou definitivamente a geografia humana nos Estados Unidos, Pfeifer concentrou-se no problema da estrutura espacial da agricultura no norte da Califórnia. Essa contribuição teórica para a geografia agrária dedicou-se à questão do local de cultivo e a sucessão de formações agrárias. Seus estudos sobre a problemática político-geográfica do "frontier" nos Estados Unidos foram de significado para os seus trabalhos posteriores no Brasil. Depois de ocupar-se com as conhecidas teses de Turner, ele analisou os parâmetros políticos, sociais e econômicos dos processos de desenvolvimento espaço-temporais do "frontier" em diferentes regiões naturais. Isto com forte abordagem sócio-geográfica sob consideração dos conflitos de interesse étnicos, culturais e sócioeconômicos que mostram a contradição entre o comportamento político em diferentes espaços.

Por pedido de Waibel, Pfeifer retornou no final de 1932 dos Estados Unidos para a Alemanha para ocupar um cargo na Universidade de Bonn onde Waibel era o sucessor de Alfred Philippson desde 1929. Pfeifer ainda conseguiu terminar sua livre docência em 1933 antes da tomada de posse dos nacional socialistas que faziam certas exigências políticas para o acesso à carreira universitária. Seu estreito vínculo com Waibel fez com que as atividades de Pfeifer durante o tempo nazista fossem influenciadas, não sendo, portanto nomeado para uma cátedra.

Pfeifer trabalhou de 1947 a 1949 em Hamburgo e em 1950 foi convidado para ir ao Brasil ao mesmo tempo foi nomeado para a cátedra de geografia na Universidade de Heidelberg, fundada por Alfred Hettner ${ }^{8}$ em 1906. Depois da longa cesura da segunda guerra mundial foi iniciada

\footnotetext{
${ }^{6}$ Faziam parte, entre outros, J.H. von Thünen, I.G. Kohl, Th.H. Engelbrecht, W. Roscher, A. Weber. F. Aereboe e C. Brinkmann.

${ }^{7} \mathrm{O}$ conceito da formação econômica era definido como uma estrutura homogênea da paisagem econômica formada por um sistema operacional agrário e pelo o objetivo da produção.

${ }^{8}$ Alfred Hettner (1859-1941), o decano da geografia alemã, é conhecido no Brasil principalmente pelas suas obras sobre os métodos da geografia (1927) e pela geografia regional comparativa (1933-35). Em sua fase inicial vacilou em sua opção de se especializar em temas da América Latina. Depois de alguns anos na Colombia ele viajou pelo sul do Brasil no fim dos anos de 1880.
} 
nova fase da pesquisa de geografia humana de Pfeifer no novo mundo - agora com foco na América Latina, principalmente no Brasil.

\section{Waibel levou o entusiasmo nas pesquisas sobre o Brasil para Heidelberg}

A estada de alguns meses no Brasil, os detalhados debates com Waibel - Pfeifer residia com Waibel em Ipanema - e o contato com os novos colegas brasileiros foram descritos por Pfeifer como mudança de rumo nas suas atividades de pesquisas. A grande excursão ao Espírito Santo, Minas Gerais e Bahia foi infelizmente atrasada, pois Waibel adoeceu e só pôde participar na Bahia. Walter Egler e Pedro Geiger foram então os acompanhantes de Pfeifer. ${ }^{9}$ Esta viagem proporcionou a Pfeifer uma primeira impressão do desenvolvimento das diversas paisagens do leste brasileiro, marcadas pelo homem. Outras excursões, no âmbito do Congresso Internacional de Geografia no Rio de Janeiro em 1956 levaram-no a Amazônia, Goiás e Minas Gerais. Em 1962 e 1965 foram realizadas excursões ao sul do Brasil, ao Planalto Central e Mato Grosso apoiadas pelo Centro de Pesquisas de Geografia do Brasil no Rio de Janeiro e pelo CNG. Acompanharam a excursão geógrafos brasileiros e, pelo lado alemão, o colaborador Gerd Kohlhepp.

Pfeifer inteirou-se intensamente com as questões básicas da geografia humana do Brasil tornando-se assim, nos anos de 1950 e 60, um dos melhores conhecedores do Brasil entre os geógrafos estrangeiros. ${ }^{10} \mathrm{Em}$ suas publicações mais importantes, ele tratou da posição do Brasil no desenvolvimento no Novo Mundo sob o ponto de vista da geografia humana (PFEIFER, 1952), analisou os fundamentos coloniais do desenvolvimento espacial do país, os ciclos econômicos dominantes em sua diferenciação regional e o significado econômico de exportação para o desenvolvimento geral. Em um trabalho sobre o mundo atlântico (1966a) ele aprofundou a descrição da situação das paisagens econômicas do Novo Mundo através de comparações com o sudeste dos Estados Unidos que ele bem conhecia. Como síntese, ele reconhece no tempo colonial uma zona homogênea de fazendas orientadas para o mercado mundial e estabelecida sobre a importação de escravos que se estendia das Carolinas nos Estados Unidos pelo Caribe até o litoral de São Paulo. Isto significou para Pfeifer "um clima social desfavorável" baseado numa constituição feudal de economia e trabalho que ainda fez efeito por muito tempo (1952, p. 96).

Estudos sobre o desenvolvimento populacional e a migração interna (1956), sobre a exploração de minérios e a indústria de ferro e aço de Minas Gerais, uma análise bem fundamentada sobre o Brasil como país em desenvolvimento sob consideração do potencial de desenvolvimento (1962a) como também uma análise da geografia urbana sobre a nova capital de Brasília (1962b) mostram a grande diferenciação temática dos seus trabalhos. Seu estudo sobre Brasília mostra também as estratégias políticas e as necessidades econômicas e relativas ao trânsito na exploração do interior. O local da nova capital, de cujas tentativas de marcação Waibel participou, deixa reconhecer a posição-chave logística de Brasília para a incorporação do interior e com isso da região da Amazônia.

\footnotetext{
${ }^{9}$ Ver o artigo de P.P. Geiger sobre a excursão na Revista Brasileira de Geografia, v.31, n.3, p. 403-442, 1951. Os diários e a correspondência de Pfeifer encontram-se no arquivo privado do autor em Tübingen.

${ }^{10}$ Ver Kohlhepp (1987/88).
} 
Paralelamente as suas extensas atividades de ensino da geografia geral, Pfeifer dedicou grande energia aos projetos de pesquisa da geografia econômica e social do Brasil. Seus contatos com colegas brasileiros e com os professores visitantes Hilgard O'Reilly Sternberg (UFRJ) em 1961 e Orlando Valverde (CNG) em 1967 na Universidade de Heidelberg (KOHLHEPP, 2013, 2017a, b) deram impulso decisivo à intensidade das pesquisas. Duas geógrafas do CNG, Regina Mousinho de Meis e Maria Novaes Pinto, também se encontravam como cientistas visitantes em Heidelberg onde se formou um centro de pesquisas geográficas sobre o Brasil na Alemanha. ${ }^{11}$ No entanto não foi possível a criação, em Heidelberg, de um centro regional interdiciplinar de estudos sobre a América Latina. Em 1962, Pfeifer foi convidado a atuar como professor visitante no Centro de Pesquisas de Geografia do Brasil da UFRJ.

Extremamente louvável foi a iniciativa de Pfeifer para publicação póstuma do manuscrito ainda não completo de Waibel sobre a colonização européia no sul do Brasil (WAIBEL, 1955) uma versão fortemente ampliada do artigo de 1949. Isto levou a trabalhos próprios de Pfeifer sobre a diferenciação do espaço econômico do sul do Brasil com a gênese de tipos de paisagens diferentes na zona limiar espanhola-portuguesa no tempo colonial e a contradição atual de regiões de pecuária nos campos da Campanha e regiões de colonização européia dos imigrantes do século XIX nas regiões florestais subtropicais do Alto Uruguai no Rio Grande do Sul (PFEIFER, 1967). O significado dos grupos sociais de camponeses, em parte de diferentes origens étnicas e com elementos tradicionais e inovadores, foi estudado com vistas ao desenvolvimento agrário.

A temática da fronteira, que ele já pesquisou nos Estados Unidos, foi examinada num estudo sobre a frente pioneira na parte norte de Mato Grosso e no norte do Paraná. Nisso ele reconheceu que nas primeiras regiões exploradas no interior do Brasil não pôde ser constatado um desenvolvimento demográfico e econômico rápido, ao contrário do que nos Estados Unidos e diferente do que nas regiões dinâmicas nas atuais fronteiras nos Estados brasileiros mencionados (1966b).

Por ocasião dos 80 anos de Waibel no ano de 1968 e lembrança do cientista que foi perseguido pelos nazistas na Alemanha, foi realizado um grande simpósio em Heidelberg sobre geografia agrária (PFEIFER, 1971), no qual foram debatidos os fundamentos metódicos e teóricos e os resultados de suas pesquisas para a geografia econômica atual.

Os professores visitantes brasileiros O'Reilly Sternberg e Valverde possibilitaram nos anos 60 que alunos de Pfeifer pudessem realizar pesquisas maiores no Brasil como Gerd Kohlhepp no sul do Brasil e Gisbert Glaser em Goiás. Como já mencionado (KOHLHEPP, 2013, 2017a) um projeto de pesquisa na Amazônia, já planejado no começo dos anos 70, pelo grupo de trabalho de Pfeifer com Orlando Valverde e colaboradores do CNG não foi realizado devido a "impedimentos nacionais de natureza político-financeira e problemas administrativos no IBGE” (2013, p. 60). Pfeifer que em 1956 pôde somente realizar uma curta excursão para a Amazônia, queria ainda participar como professor emérito nos estudos sobre a Amazônia.

Em Heidelberg, Pfeifer tinha dado ênfase à criação da pesquisa geográfica alemã sobre o Brasil além de iniciar, juntamente com Wilhelm Lauer (Bonn), um programa interdisciplinar de pesquisas alemão-mexicano, financiado pela Associação Alemã de Pesquisas (DFG) do qual par-

\footnotetext{
${ }^{11}$ Nas "Contribuições para a Geografia do Brasil", os trabalhos foram reunidos em uma publicação conjunta dos cientistas visitantes com Valverde, Glaser e Kohlhepp (GLASER, 1971).
} 
ticiparam uma série de geógrafos alemães e alunos de Pfeifer de Heidelberg. Outrossim, o relançamento da "Geographische Zeitschrift" (Revista Geográfica) em 1963, fundada por Hettner e, sem dúvida, um dos órgãos publicitários mais importantes da geografia alemã também foi uma iniciativa de Pfeifer.

As antigas publicações de Pfeifer, infelizmente quase que exclusivamente em idioma alemão, foram naturalmente úteis como base científica da situação geográfica e da diferenciação regional do Brasil para a geografia científica alemã. As repartições e o meio público naquela época, na Alemanha, tinham poucos conhecimentos sobre o Brasil, o maior país tropical do mundo. Devido aos anos de guerra, Pfeifer iniciou suas pesquisas sobre o Brasil relativamente tarde. No entanto, Pfeifer motivou e despertou interesse de seus colaboradores Gerd Kohlhepp e Gisbert Glaser para a pesquisa geográfica sobre o Brasil. Glaser optou pela UNESCO não dando prosseguimento às suas pesquisas sobre o Brasil. ${ }^{12}$

A presença do professor visitante Hilgard O'Reilly Sternberg em Heidelberg levou a um convite a Kohlhepp no sentido de realizar seus trabalhos de doutoramento no Brasil. A tese sobre a geografia industrial do nordeste de Santa Catarina (KOHLHEPP, 1968) ${ }^{13} \mathrm{com}$ trabalhos de campo de 1962/63 levou a abordagens metódicas novas nesta quase desconhecida parte da geografia humana. Ficou claro que a indústria com ramos específicos e muito diferenciados estava baseada nos impulsos das múltiplas fases da mão de obra especializada, sobretudo dos imigrantes alemães, na qualidade da mão de obra local e regional como também no capital local. Este estudo foi o ponto de partida para uma série de trabalhos sobre a geografia e a história econômica do Sul do Brasil e a estrutura econômica do Brasil.

O projeto de pesquisa "Colonização do Norte do Paraná" de Kohlhepp com intensos trabaIhos de campo no ano 1970 foi concluída como tese de livre docência em 1972. A publicação (1975) foi traduzida para o português em 2014. ${ }^{14}$ No estudo foram analisados processos de geografia econômica e social no desenvolvimento de uma zona pioneira na periferia dos trópicos sob a influência da plantação do café, o avanço da frente pioneira, os grupos e classes sociais e sua mobilidade espacial e social. O desenvolvimento dessa região que nos anos de 1960 tornou-se a região de plantação do café de maior importância mundial, foi influenciada tanto pelas intervenções do Estado com os programas de erradicação do café e legislação social como também pelas consequências severas das repetidas geadas. Foram considerados os problemas de meio ambiente por devastação de florestas, políticas de diversificação do uso da terra, transformações do sistema agrário e a reorganização do mercado mundial do café.

No ano de 1972, com a aposentadoria de Pfeifer e o convite para Gerd Kohlhepp de assumir a cátedra de geografia humana na Universidade de Frankfurt, ${ }^{15}$ terminou a pesquisa da geografia sobre o Brasil em Heidelberg.

\footnotetext{
12 Glaser pesquisou sobre a exploração de estanho em Rondônia (1969) e sobre a pecuária no Brasil Central (GLASER, 1971).

${ }^{13}$ Vide recensão de Orlando Valverde na Revista Brasileira de Geografia, v.31, n.2, p. 81-85, 1969.

14 O trabalho foi publicado em Maringá (KOHLHEPP, 2014).

${ }^{15} \mathrm{Na}$ Alemanha, a ascensão profissional de livre docência para professor titular não é possível na própria universidade, mas somente por nomeação para uma outra universidade.
} 


\section{Pesquisas geográficas sobre o Brasil em Frankfurt e concentração no Centro de Pesquisas sobre a América Latina na Universidade de Tübingen a partir de 1978}

Depois da conclusão do projeto no Paraná foram iniciadas as atividades de Kohlhepp em Frankfurt (1972-78) com pesquisas sobre a Amazônia em coordenação com colegas e universidades brasileiras, mas inicialmente como pesquisas individuais. Como o programa de pesquisas sobre a Amazônia planejado ainda em Heidelberg com Valverde e com o CNG não pôde ser realizado, estudos com um espectro amplo de tópicos amazonenses foram realizados. O ponto de partida dos trabalhos era composto pela análise da colonização agrária na Transamazônica (1976), estratégias estatais para Amazônia Legal, a mineração e o desenvolvimento de assentamentos na parte leste da região amazônica, conflitos de interesse na frente pioneira com consequências para a população indígena como também avaliação de processos sócio-econômicos na periferia à luz de alternadas estratégias da política agrária (1979). ${ }^{16}$

Com a sua nomeação como professor titular de geografia econômica e social na reconhecida Universidade de Tübingen em 1978 foi possível para Kohlhepp criar um "Centro de Pesquisas sobre a América Latina" na pesquisa, no qual o Brasil ocupava o enfoque principal. O seu antecessor, Herbert Wilhelmy, um dos geógrafos mais renomados da Alemanha, já tinha a América Latina como um dos pontos centrais de seus trabalhos. ${ }^{17}$

Com a organização de um grupo de trabalho com bons conhecimentos do idioma português foi iniciada uma série de projetos de pesquisas no Brasil. No Brasil foi criada uma rede de contatos com colegas também de outras disciplinas. Os estudos na região amazônica referiam-se aos planejamentos estatais para programas de desenvolvimento regional, a colonização agrária dirigida e espontânea (1984), a pecuária extensiva no Pará e em Mato Grosso (1987a), o Programa Polonoroeste em Rondônia e uma evaluação do Programa Grande Carajás e suas consequências sócio-econômicas (1987b). No ano de 1986 foram realizados em Tübingen dois congressos internacionais sobre a Amazônia e problemas ecológicos na América Latina, com forte participação brasileira.

Além disso, foram realizadas pesquisas de geografia agrária no sul do Brasil, mais precisamente na região do Alto-Uruguai, no oeste e no norte do Paraná (1987c). Trabalhos sobre a colonização agrária dos Suábios de Danúbio nos campos limpos do Paraná, a migração da população rural desempregada e de baixa renda para o leste do Paraguai e Mato Grosso estão relacionados com trabalhos anteriores sobre a colonização agrária no Paraná. As mudanças da estrutura agrária e do desenvolvimento regional no norte do Paraná foram analisadas em um estudo comparativo a 1970 num espaço de tempo de 20 anos (1991). As consequências sócioeconômicas e ecológicas da usina hidrelétrica e da represa de Itaipu foram examinadas numa monografia (1987d) e em outros estudos adicionais.

Desde o início dos anos de 1980 foi formado considerável grupo de doutorandos e colabo-

\footnotetext{
${ }^{16}$ Aqui só podem ser mencionadas as publicações mais importantes, isto é, sobretudo os trabalhos publicados em português (vide Referências).

17 Em 1952, Wilhelmy relatou sobre cidades da América do Sul e do Brasil, atualizando este tema nos anos de 1980 (WILHELMY \& BORSDORF, 1984/1985). Além de artigos sobre a Amazônia (1966), uma contribuição orientadora sobre o Grande Pantanal (1958) é muito conhecida e foi traduzida em Tübingen e publicada nos Cadernos do NERU em Cuiabá (WILHELMY, 1997).
} 
radores em Tübingen com enfoque na pesquisa sobre o Brasil. Com relação à publicação de teses de doutorado selecionadas foi criada em 1986 por Gerd Kohlhepp uma nova série de publicações: "Contribuições Tübingenses para a pesquisa geográfica sobre a América Latina" (TBGL). ${ }^{18}$ Para cada trabalho em idioma alemão havia um resumo detalhado em portugues. As melhores teses de mestrado ${ }^{19}$ eram publicadas em série própria (KAGIT).$^{20}$

Com teses sobre duas das principais regiões de trabalho no sul do Brasil (LÜCKER, 1986: desenvolvimento agrário na região do Alto Uruguai) e Amazônia (COY, 1988) foi iniciada uma longa série de teses de doutorandos alemães e brasileiros que realizaram seus trabalhos de campo no Brasil, defendendo suas teses em Tübingen. A primeira tese de um doutorando brasileiro em Tübingen foi o trabalho de geografia urbana de Marcelo Lopes de Souza sobre a segregação do espaço social e conflitos sociais na região metropolitana do Rio de Janeiro (SOUZA, 1993). Uma lista das principais teses, algumas até premiadas, consta da parte final desta contribuição (depois das Referências). Deve ser mencionado que as teses de doutorandos brasileiros foram apresentadas em idioma alemão.

As transformações do espaço social e econômico em regiões do Brasil Central foram tema de um projeto no qual foram estudados os impactos sócio-econômicos e espaciais da política de modernização agrícola e os conflitos de interesse entre os diferentes grupos sociais em regiões periféricas do Brasil. Exemplos de caso foram a agricultura modernizada no sul do Centro-Oeste (Dourados/MS e Rio Verde/GO) e a diferenciação de uma nova frente pioneira baseada em colonização particular no norte de Mato Grosso (COY \& LÜCKER, 1993).

Depois da sua tese sobre desenvolvimento regional em Rondônia, o foco das pesquisas de Martin Coy concentrou-se primeiramente nas frentes pioneiras e cidades pioneiras no norte de Mato Grosso (COY, 1991, 1996) e sua tese de livre docência sobre o desenvolvimento urbano e as possibilidades da mudança estrutural sustentável de Cuiabá (entre outros, 1994). Estudos adicionais tratavam da modernização no espaço rural entre a globalização e a regionalização bem como da expansão do cultivo da soja $(2001,2003)$ e da ecologia política de frentes pioneiras no sudoeste da Amazônia (2005).

Nos anos de 1990, um abrangente projeto de pesquisas em Mato Grosso ocupou quase que inteiramente o Centro de Tübingen. Juntamente com um grupo multidiciplinar de colegas do Núcleo de Estudos Rurais e Urbanos (NERU - UFMT) em Cuiabá, foram estudados a "Estrutura sócio-econômica e dinâmica dos impactos ambientais na Bacia do Alto Rio Paraguai, Mato Grosso". O projeto fazia parte da cooperação científico-tecnológica em pesquisa sobre ecossistemas tropicais entre o Brasil e a Alemanha (CNPq - BMFT). Realizado no âmbito do programa bilateral de pesquisa "Studies on Human Impacts on Forests and Floodplains in the Tropics (SHIFT), o projeto teve como objetivo analisar as estruturas sócio-econômicas, suas transformações e suas severas implicações ambientais no Pantanal e na Bacia do Alto Rio Paraguai. O Programa SHIFT foi uma das atividades científicas de maior significado dentro da cooperação bilateral Brasil-

\footnotetext{
18 TBGL é a abreviatura alemã da série. De 1986 a 2007 foram publicados 31 volumes. As teses de doutoramento mais importantes e publicadas nesta série são mencionadas depois das Referências.

${ }^{19} \mathrm{Na}$ Alemanha, tradicionalmente o título acadêmico adquirido no término do estudo universitário chamava-se Diplom. Hoje chama-se Master (M.A., M.Sc.) o que corresponde ao mestrado.

${ }^{20}$ A série KAGIT continha, entre outros, com trabalhos sobre garimpos em Mato Grosso, turismo no Pantanal e na região Gramado-Canela/RS, o Projeto Prodeagro em Mato Grosso, política regional e desenvolvimento sustentável no Amapá, segregação sócio-espacial no Rio de Janeiro (Barra da Tijúca), desenvolvimento high tech em Blumenau/SC.
} 
Alemanha.

O Projeto em Mato Grosso, cujos trabalhos de campo foram coordenados por Paulo Speller (Cuiabá) e Martin Coy (Tübingen) e do qual participaram 25 cientistas dos dois lados, tinha o objetivo teórico de fornecer uma contribuição à análise das relações homem-meio ambiente no contexto da ecologia dos trópicos. A composição interdisciplinar do grupo de pesquisadores fez necessária a elaboração de metodologia interdisciplinar. Numa cooperação exemplar foi elaborado um Diagnóstico Sócio-econômico em 12 volumes ${ }^{21}$ com uma série de mapas temáticos, o que permitiu a regionalização dos potenciais de conflitos sócio-econômicos e ecológicos. O diagnóstico foi utilizado como base de debate de concepções de desenvolvimento regional ecologicamente adaptadas e sustentáveis com a sociedade civil e deveria servir como propostas aos órgãos executivos estaduais. Em uma segunda fase foi criado amplo espectro de estudos de caso, apresentados em diversas teses de doutorado e de mestrado em Cuiabá e pelos participantes alemães em Tübingen depois de alguns anos de estada na região de trabalho.

Os resultados dos projetos, além de apresentados em coletâneas com contribuições dos colaboradores (NERU,1994; KOHLHEPP,1995; KOHLHEPP \& COY,1998,1999) e nos Proceedings de diversos Workshops do complexo Programa SHIFT, foram publicados sobretudo em teses de doutoramento. Do lado de Tübingen eram, entre outros, teses ${ }^{22} \mathrm{com}$ os seguintes temas: Modificações no uso da terra na agricultura modernizada na Chapada dos Parecis (BLUMENSCHEIN, 2001), planejamento e implementação de áreas de proteção na região do Pantanal (RÖPER, 2001), vulnerabilidade e estratégias de sobrevivência de pequenos colonos no hinterland de Cáceres (NEUBURGER, 2002), conflitos por recursos naturais e estratégias de proteção de áreas para os povos indígenas (PASCA, 2004) e desenvolvimento urbano dos centros regionais Cáceres e Rondonópolis (FRIEDRICH, 1999).

Trabalhos da geografia humana sobre o Brasil foram realizados nesta fase, além de Tübingen somente em poucos Institutos de Geografia em universidades alemãs, sobretudo em Kiel e em casos individuais em Marburg, Bonn e Passau.

Nos anos de 1990 foram intensificadas as atividades do Governo Brasileiro relativas à diminuição da devastação de florestas na Amazônia com cooperação internacional. O "Programa Piloto Internacional para a proteção das florestas tropicais do Brasil " (PPG7) correspondeu à mais ampla tentativa feita, no sentido de juntar atores globais - Banco Mundial, países do G7, União Européia - com o Governo Brasileiro e atores nacionais, regionais e locais, em um Programa com objetivos de desenvolvimento regional sustentável estabelecidos na Agenda 21 da Conferência de Meio Ambiente e Desenvolvimento (UNCED - "ECO 92") no Rio de Janeiro em 1992 (KOHLHEPP, 2001).

Desse modo surgiram desafios especiais para a ciência geográfica, não somente por publicação e debate de resultados de pesquisas, mas também através de grêmios e comissões científicos referentes à Amazônia. A comissão científica independente, internacional e multidisciplinar de peritos com 11 membros (International Advisory Group - IAG) do Programa Piloto tinha a incumbência de analisar criticamente o Programa e contribuir com sugestões para o melhoramento das estratégias e questionar problemas com os responsáveis. No IAG a geografia conseguiu mais

\footnotetext{
${ }^{21}$ Os volumes produzidos pelos colaboradores do projeto de Tübingen e Cuiabá, vide CPAL \& NERU (1994/95).

22 Vide Referências neste trabalho.
} 
destaque com a nomeação de Bertha K. Becker, Hervé Théry e Gerd Kohlhepp como membros e periodicamente dirigentes do grupo.

Num congresso internacional e interdisciplinar "Brasil: modernização e globalização" da Associação Alemã de Pesquisas sobre a América Latina (ADLAF) em Tübingen em 1998 com grande participação brasileira - a palestra de abertura foi proferida por Celso Lafer - foram debatidos temas como os 500 anos do Brasil, a integração supranacional e a identidade regional, a questão urbana, o meio ambiente e o desenvolvimento regional (KOHLHEPP, 2001).

Numa rede de pesquisa multilateral e interdisciplinar com os colegas Bertha K. Becker (Rio de Janeiro), Antony Hall (Londres), Hervé Théry (Paris), Roberto Smeraldi (São Paulo) e Felipe Mansilla (La Paz) foi debatida a elaboração da análise dos processos sócio-econômicos e ecológico-políticos no Brasil e na Bolívia diante da necessidade de desenvolvimento sustentável na Amazônia. Os primeiros resultados foram publicados em 2005 (COY \& KOHLHEPP, 2005).

O Centro de Pesquisas em Tübingen contava com cooperação e contatos com uma série de universidades brasileiras ${ }^{23}$ e de outros países lationoamericanos. A partir de 1988, o DAAD (Serviço Alemão de Intercâmbio Acadêmico) financiou um programa de intercâmbio integrado para estudantes de graduação (chamado mais tarde de Programa Unibral) com a UFRJ do qual mais de 80 estudantes de Tübingen e do Rio de Janeiro participaram até 2005. Antes de iniciar suas viagens, os estudantes podiam frequentar um curso de idioma. Na universidade parceira participaram nos cursos de geografia, reconhecidos por Tübingen e pelo Rio, durante um ano letivo. Assim também os estudantes alemães, com os conhecimentos linguísticos necessários, tinham a possibilidade de colaborar em projetos de pesquisas e na realização de trabalhos de campo no Brasil para fins de mestrado. Antes da Unibral, estudantes brasileiros eram financiados com meios do Centro, da Universidade e do fundo dos ex-bolsistas alemães. O bom ambiente do Instituto mostrou-se também pela realização de diversos casamentos entre estudantes alemães e brasileiros.

Além dos cursos de geografia humana e geografia física, o Instituto de Geografia de Tübingen ofereceu o curso "geografia dos países em desenvolvimento", a partir de 1994. Na Alemanha somente Tübingen e Berlim ofereciam estes cursos, mesmo sendo o Oriente o enfoque em Berlim. Em Tübingen, o currículo concentrou-se em aspectos de geografia da economia mundial, da geografia econômica e social sobre países emergentes e em desenvolvimento, com enfoque regional principalmente na América Latina. O objetivo era oferecer uma formação voltada à aplicabilidade profissional com sólida fundamentação teórica e pautada em metodologia e conhecimentos regionais específicos. Os estudos procuravam atender as multifacetadas exigências do trabalho na cooperação internacional. Para tal eram oferecidas excursões anuais, de duração de algumas semanas para o Brasil e outros países latinoamericanos. Além das excursões, eram realizados diversos estágios práticos de trabalhos de campo em diversas regiões do Brasil.

Depois de completados seus trabalhos de doutorado em Tübingen, uma série de geógrafos brasileiros e alemães tornaram-se professores em universidades no Brasil: Marcelo J. Lopes de Souza (UFRJ), Cicilian L. Löwen Sahr (UEPG, Ponta Grossa), Ivo M. Theis (FURB, Blumenau), Dieter R. Siedenberg (UNIJUI), Erica Karnopp (UNISC), Wolf-Dietrich Sahr (UFPR) e Karl Henkel

\footnotetext{
23 Tübingen tinha acordos ou contatos aprofundados com as seguintes universidades brasileiras e com colegas da geografia: UFRJ, UFF, USP, UnB, UFMT, UNIR, UFPA, UFBA, UFPE, UFPR, UEM Maringá, UFSC, FURB Blumenau, UNISC, UFRGS, PUCRS e com outras instituições científicas, p.ex. INPA.
} 
(UFPA). Outros doutorandos que trabalharam sobre temas brasileiros, são hoje professores na Alemanha, na Áustria e no Canadá. Os formados em mestrado trabalham em organizações estatais, privadas ou instituições eclesiásticas da cooperação de desenvolvimento ou como consultores no Brasil.

\section{Considerações finais: Novos rumos para as pesquisas sobre o Brasil}

Depois que Gerd Kohlhepp, pertencente à geração dos "netos de Waibel" tornou-se professor emérito em 2005, terminou a atividade do "Centro de Pesquisas de Geografia sobre a América Latina" na Universidade de Tübingen. O enfoque "Brasil" não teve continuidade no Instituto de Geografia da Universidade de Tübingen e consequentemente os programas de intercâmbio com o Brasil e a Argentina não foram prolongados. Algumas das teses de doutoramento em andamento ainda foram orientadas por alguns anos por Gerd Kohlhepp e Rainer Rothfuß, que defendeu sua tese de doutorado também em Tübingen (ROTHFUSS, 2006) sobre um tema de geografia urbana, com estudos de caso no Brasil. A última tese de doutoramento orientada por Gerd Kohlhepp relativa ao Brasil trata do modelo de planejamento urbano participativo em Porto Alegre (MORORÓ, 2014).

Kohlhepp ainda elaborou alguns estudos nos últimos anos: desenvolvimento regional na Amazônia (2007), estudo sobre a produção de etanol e biodiesel em São Paulo (2008), o significado da indústria para o desenvolvimento do nordeste de Santa Catarina como estudo comparativo à tese de quase 50 anos atrás (2010) como também sobre a temática do "frontier" (COY, KLINGLER e KOHLHEPP,2017). Em coletâneas multidisciplinares foram publicados fundamentos geográficos sobre o Brasil $(2003,2010)$. Homenagens a geógrafos brasileiros como Orlando Valverde e Hilgard O`Reilly Sternberg, que muito fizeram pela cooperação Brasil-Alemanha na geografia, foram publicadas recentemente $(2017 a, b)$.

Gerd Kohlhepp atuou como perito em dois grandes programas de pesquisas AlemanhaBrasil: WAVES (Water Availability of Ecosystems and Society in the Northeast of Brazil: 19962001) e CARBIOCIAL (Sequestração de carbono, biodiversidade e estruturas sociais no sul da Amazônia: 2011 - 16). A edição da "Geographische Zeitschrift " (co-editor de 1977 - 2002) e de outras revistas geográficas especializadas como diversas séries de publicações como "Erdkundliches Wissen" ("O saber geográfico": 1983 - 2007) entre outros, foi passada para jovens colegas. Como membro do Conselho Editorial, Kohlhepp ainda atua em alguns órgãos de publicação da geografia do Brasil. Para o autor é uma honra ser membro estrangeiro da Associação Brasileira de Ciências, continuando a proferir palestras no Brasil e participar de congressos e simpósios internacionais e em universidades brasileiras.

Martin Coy, colaborador de muitos anos do Centro, atua desde 2003 em Innsbruck/Áustria. Em 2006 foi nomeado para a nova cátedra de Geografia Aplicada e Pesquisas de Sustentabilidade daquela Universidade formando um grande grupo de trabalho, que exerce a pesquisa sobre o Brasil como enfoque principal. Colaboradores de Tübingen como Martina Neuburger $(2002,2004)$ e Frank Zirkl (2007), que fizeram seu doutoramento em Tübingen e alguns outros jovens geógrafos puderam dar continuidade aos seus trabalhos em Innsbruck. 
Como a visão geral dessa contribuição termina por volta do ano de 2005, somente será possivel discorrer brevemente sobre universidades alemãs que hoje realizam pesquisas sobre o Brasil em geografia humana. Em Kiel, junto a Rainer Wehrhahn e seu grupo de trabalho é dado enfoque regional às pesquisas sobre o Brasil. Martina Neuburger (Hamburgo), Dörte Segebart (Berlim) e Eberhard Rothfuß (Bayreuth) são os demais colegas que trabalham sobre Brasil, em temas da geografia humana.

Para o autor desta contribuição é uma grande satisfação ter tido a oportunidade de aplicar a idéia de Leo Waibel na continuidade e intensidade dos contatos científicos entre geógrafos no Brasil e na Alemanha. Com pesquisas próprias e as do grupo de colaboradores puderam ser intensificadas na Alemanha as pesquisas da geografia humana do Brasil num ramo temático muito amplo. Entrementes, com colegas mais jovens, novos temas e métodos de trabalho poderão acompanhá-los em seus caminhos. 
Referências

BELL, S. Prelude to Brazil: Leo Waibel's American career as a displaced scholar. Geographical Review, v. 106, n.1, p. 5-27, 2016.

BERNARDES, N. Leo Waibel. Revista Brasileira de Geografia, vol.14, n.2, p. 199-201,1952.

BERNARDES, N. Address delivered on the occasion of the closing session of the Second LatinAmerican Regional Conference of the IGU, Rio de Janeiro 1982. IGU-Bulletin, n.33, p. 1222, 1983.

COY, M. The frontier of North Mato Grosso between soybean production, timber extraction and gold mining. In: KLEINPENNING, J.M.G. (coord.): The incorporated drive. Examples from Latin America. Nijmegen Studies in Development and cultural change, vol.8, p. 40-58, 1991.

COY, M. Transformação sócio-ambiental do espaço urbano e planejamento em Cuiabá. In: Cadernos do NERU, vol.3, p. 131-174, Cuiabá, 1994/95.

COY, M. Cidades pioneiras e desenvolvimento sustentável na Amazônia brasileira. Transformação sócio-econômica e desafios para o planejamento nas frentes pioneiras. Geosul, v.10, n.19/20, p. 51-67, 1996.

COY, M. Entre a globalização e a regionalização. Efeitos da modernização, conflitos de interesse e fatores determinantes do desenvolvimento regional sustentável no espaço rural brasileiro. In: KOHLHEPP, G. (coord.), Brasil - modernização e globalização. Bibliotheca IberoAmericana, vol.80, Frankfurt am Main: Vervuert, 2001, p. 259-271.

COY, M. Desenvolvimento regional no sudoeste da Amazônia. Mudança espacial sócioeconômica na periferia brasileira entre globalização e sustentabilidade (Regionalentwicklung im südwestlichen Amazonien). In: KOHLHEPP, G. (coord.): Brasil. Tübingen: Attempto, 2003, p. 215-238.

COY, M. Between globalization and regionalization. The political ecology of pioneer fronts in the South-West of the Amazon. In. HALL, A.(coord.): Global impact, local action: New environmental policy in Latin America. London: Univ. of London, 2005, p. 13-35.

COY, M.; LÜCKER, R. O Centro Oeste do Brasil. Transformação da geografia econômica e social de uma região agrária periférica (Der brasilianische Mittelwesten). Contribuições Tübingenses para a Pesquisa Geográfica sobre a América Latina (TBGL), vol.9, Tübingen: Inst. de Geografia, 1993.

COY, M.; KOHLHEPP, G. (coords.). Amazônia sustentável. Desenvolvimento sustentável entre políticas publicas, estratégias inovadoras e experiências locais. Rio de Janeiro: Garamond, 2005 (= TBGL, vol.28, Tübingen, 2005).

COY, M., KLINGLER, M., KOHLHEPP, G. De frontier até pós-frontier: regiões pioneiras no Brasil dentro do processo de transformação espaço-temporal e sócio-ecológico. Confins. Revista franco-brasileira de geografia, n.30, p. 1-48, 2017.

CPAL \& NERU (eds.). Diagnóstico do projeto de pesquisa "Estrutura sócio-econômica e dinâmica dos impactos ambientais na Bacia do Alto Rio Paraguai, Mato Grosso", 12 
vols. Cuiabá, Tübingen, 1994/95.

GLASER, G. (coord.). Contribuições para a geografia do Brasil (Beiträge zur Geographie Brasiliens). Heidelberger Geogr. Arbeiten (HGA), vol.34, Heidelberg: Inst. de Geografia, 1971.

KOHLHEPP, G. Geografia industrial do Nordeste de Santa Catarina (Industriegeographie des nordöstlichen Santa Catarina). HGA, vol.21, Heidelberg: Inst. de Geografia, 1968 (tese de doutoramento).

KOHLHEPP, G. Colonização agrária no Norte do Paraná. Processos geoeconômicos e sociogeográficos de desenvolvimento de uma zona pioneira tropical periférica do Brasil sob a influência da plantação do café. Maringá: EdUEM, 2014 (= Agrarkolonisation in Nord-Paraná. Wiesbaden: Steiner, 1975).

KOHLHEPP, G. Planejamento e situação atual dos projetos estatais de colonização agrária de pequenos colonos na Transamazônica (Planung und heutige Situation staatlicher kleinbäuerlicher Kolonisationsprojekte an der Transamazônica). Geographische Zeitschrift, v.64, n.3, p. 171-211, 1976.

KOHLHEPP, G. A antítese problemática à reforma agrária: Colonização agrária na Amazônia (Brasiliens problematische Antithese zur Agrarreform: Agrarkolonisation in Amazonien). In: ELSENHANS, H. (coord.): Reforma agrária no Terceiro Mundo (Agrarreform in der Dritten Welt). Frankfurt am Main, New York: Campus, p. 471-504, 1979.

KOHLHEPP, G. Development planning and practices of economic exploitation in Amazonia. Recent trends in spatial organization of a tropical frontier region in Brazil (1966-1981). In: SIOLI, $\mathrm{H}$. (coord.): The Amazon. Limnology and landscape ecology of a mighty tropical river and its basin. Monographiae Biologicae, vol.56, Dordrecht, Boston, Lancaster: Dr. W. Junk Publishers, 1984, p. 649-674.

KOHLHEPP, G. Amazônia. Desenvolvimento regional no foco de conflitos entre interesses econômicos e necessidades sociais e ecológicas (Amazonien. Regionalentwicklung im Spannungsfeld ökonomischer Interessen sowie sozialer und ökologischer Notwendigkeiten). Problemräume der Welt, vol. 8, Köln: Aulis, 1987a.

KOHLHEPP, G. Problemas de planejamento regional e de desenvolvimento regional na área do Programa Grande Carajás no Leste da Amazônia. In: KOHLHEPP, G.; SCHRADER, A. (coords.): Homem e natureza na Amazônia. TBGL, vol.3, Tübingen: Inst. de Geografia,1987b, p. 313-345.

KOHLHEPP, G. (coord.). Brasil. Contribuições para a pesquisa da estrutura e de desenvolvimento regional (Brasilien. Beiträge zur regionalen Struktur- und Entwicklungsforschung). TBGL, vol.1, Tübingen: Inst. de Geografia, 1987c.

KOHLHEPP, G. Itaipú. Basic geopolitical and energy situation - socio-economic and ecological consequences of the Itaipú dam and reservoir on the Rio Paraná (Brazil/Paraguay). GTZ Division, 21, Ecology and Environmental Protection. Braunschweig, Wiesbaden: Vieweg, 1987d.

KOHLHEPP, G. Gottfried Pfeifer (20.1.1901 - 6.7.1985). In: Geographisches Taschenbuch. Stuttgart: Steiner, 1987/1988, p. 133-157. 
KOHLHEPP, G. Mudanças estruturais na agropecuária e mobilidade da população rural no Norte do Paraná. Revista Brasileira de Geografia, v. 53, n.2, p. 79-94, 1991.

KOHLHEPP, G. (coord.). Relações homem-meio ambiente na região do Pantanal em Mato Grosso. Contribuições para a pesquisa geográfica aplicada do meio ambiente (Mensch-Umwelt-Beziehungen in der Pantanal-Region von Mato Grosso). TBGL, vol.12, Tübingen: Inst. de Geografia, 1995.

KOHLHEPP, G. Estratégias da política ambiental e regional para a proteção das florestas tropicais no Brasil. O Programa Piloto Internacional e seus atores em direção ao desenvolvimento sustentável? In: KOHLHEPP, G. (coord.): Brasil - modernização e globalização. Bibliotheca Ibero-Americana, vol. 80, Frankfurt am Main: Vervuert, 2001, p. 209 - 234.

KOHLHEPP, G. (coord). Brasil. País em desenvolvimento ou potência tropical do séc. 21? (Brasilien. Entwicklungsland oder tropische Großmacht des 21). Tübingen: Attempto, 2003.

KOHLHEPP, G. Desenvolvimento regional na Amazônia Brasileira. Estratégias de ordenamento territorial e conflitos entre interesses econômicos e uso sustentável dos recursos naturais nas florestas tropicais. In: PASSOS, M.M. BR-163 - De estrada dos colonos a corredor de exportação. Maringá: Ed. Massoni, 2007, p. 15-62.

KOHLHEPP, G. Análise da situação da produção de etanol e biodiesel no Brasil. Estudos Avançados, v.23, n. 68 , p. $223-253,2010$ a.

KOHLHEPP, G. A contribuição da indústria para o desenvolvimento regional no Nordeste de Santa Catarina. In: RENAUX, M.L.: Colonização e indústria no Vale do Itajaí. O modelo catarinense de desenvolvimento. $2^{\mathrm{a}}$.ed., Florianópolis: Instituto Carl Hoepcke, 2010b, p. 345388.

KOHLHEPP, G. A importância de Leo Waibel para a geografia brasileira e o início das relações científicas entre o Brasil e a Alemanha no campo da geografia. Revista Brasileira de Desenvolvimento Regional, v.1, n.2, p.29-75, 2013.

KOHLHEPP, G. Orlando Valverde (1917-2006). Um geógrafo brasileiro de renome internacional entusiasta e entusiasmante. In: SUERTEGARAY, D.M.A. et al. (org.), Orlando Valverde. 0 geógrafo e sua obra. Porto Alegre: Geociências/ UFRGS, 2017a , p. 157-188.

KOHLHEPP, G. Hilgard O`Reilly Sternberg, um pioneiro nas pesquisas das questões ambientais no Brasil. Espaço Aberto. Revista do Programa de Pós-Graduação em Geografia da UFRJ, v.7, n.1, p. 7-21, 2017b.

KOHLHEPP, G. et al. (eds.) Brasil hoje. Espaço geográfico, política, economia, cultura (Brasilien heute. Geographischer Raum, Politik, Wirtschaft, Kultur). Bibliotheca Ibero-Americana, vol.134, Frankfurt am Main: Vervuert, 2010.

KOHLHEPP, G; COY, M. (coords.). Relações homem-meio ambiente e desenvolvimento sustentável no Terceiro Mundo (Mensch-Umweltbeziehungen und nachhaltige Entwicklung in der Dritten Welt). TBGL, vol.15, Tübingen: Inst. de Geografia, 1998.

KOHLHEPP, G.; COY, M. Socio-economic structure and environmental impact in the Upper River Paraguai Basin. A synthesis of project results. - In: BMBF (ed.): Proceedings at the Third 
SHIFT-Workshop, Manaus 1998, Geesthacht: GKSS, 1999, p. $531-547$.

MORORÓ, R.R. O mito democrático de Porto Alegre. Contradições e realidade de um "modelo de planejamento" participativo (Der demokratische Mythos Porto Alegre. Widersprüche und Wirklichkeit eines partizipativen "Planungsmodells"). Bürgergesellschaft und Demokratie, vol.45.Wiesbaden: Springer VS, 2014.

NERU (coord.). Ambiente: uma abordagem sócio-econômica. Cadernos do NERU, vol.3, Cuiabá, 1994.

NEUBURGER, M. Smallholder vulnerability in degraded areas. The political ecology of pioneer frontier processes in Brazil. Geographische Zeitschrift. Special issue, p. 58-72, 2004.

PFEIFER, G. A posição do Brasil no desenvolvimento do Novo Mundo do ponto de vista da geografia humana (Brasiliens Stellung in der kulturgeographischen Entwicklung der Neuen Welt. Kolonialzeit). Erdkunde, v.6, n.2/3, p. 85-103, 1952.

PFEIFER, G. A população urbana e rural no Brasil e a migração interna (Städtische und ländliche Bevölkerung in Brasilien und die Binnenwanderungsbewegung). Geographisches Taschenbuch, 1956/57, p. 392-402.

PFEIFER, G. Brasil, um país em desenvolvimento (Brasilien als Entwicklungsland). Westfälische Geogr. Studien, v.15, Münster: Inst. de Geografia, 1962a, p.125-194.

PFEIFER, G. Brasília. In: LEIDLMAIR, A. (ed.): Hermann von Wissmann-Festschrift (publicação comemorativa), Tübinger Geogr. Studien, Sonderband 1, Tübingen: Inst. de Geografia, 1962b, p. 289-320.

PFEIFER, G. Mundo Atlântico. Problemas de formação de paisagens culturais no Novo Mundo, o Brasil como exemplo (Atlantische Welt). Würzburger Geogr. Arb., vol.18, Würzburg: Inst. de Geografia, 1966a.

PFEIFER, G. Observaciones a lo largo de las nuevas fronteras de colonización en Paraná y Mato Grosso. In: UGI (ed.): Conf. Regional Latino-Americana. T.1.La geografía y los problemas de población. México, 1966b, p.314-328.

PFEIFER, G. Contrastes no Rio Grande do Sul: Campanha e Alto Uruguai (Kontraste in Rio Grande do Sul: Campanha e Alto Uruguai). Geographische Zeitschrift, v.55, n.3, p. 163-206, 1967.

PFEIFER, G. (Coord.). Simpósio da Geografia Agrária (Symposium zur Agrargeographie). Heidelberger Geogr. Arb. vol.36, Heidelberg: Inst. de Geografia, 1971.

PFEIFER, G.; KOHLHEPP, G. (coord.). Leo Waibel, pesquisador e planejador no Brasil (Leo Waibel als Forscher und Planer in Brasilien). Quatro artigos (Trad. G. Kohlhepp). Erdkundliches Wissen, vol.71. Wiesbaden, Stuttgart: Steiner, 1984.

ROTHFUSS, R. Transnational City Networks as an Instrument of Inter-City Cooperation in the age of global interconnectedness: The Euro-Latin American City Network URB-AL. Tübingen, 2006 (tese de doutoramento) (http://hdl.handle.net/10900/48986). 
VALVERDE, O. Contribuição de Leo Waibel à geografia brasileira. Revista Brasileira de Geografia, v.30, n.1, p. 74-83, 1968.

WAIBEL, L. O que aprendi no Brasil. Revista Brasileira de Geografia, v.12, n.3, p. 419-428, 1950.

WAIBEL, L. A colonização européia no sul do Brasil (Die europäische Kolonisation Südbrasiliens). Organização e redação de G. Pfeifer. Colloquium Geographicum, vol.4, Bonn: Ferd. Dümmler, 1955.

WILHELMY, H. O Grande Pantanal de Mato Grosso. Uma contribuição à geografia regional das planícies aluviais nos trópicos. Cadernos do NERU, vol.6 (Ecodesenvolvimento), p.99-156, Cuiabá, 1997.

WILHELMY, H.; BORSDORF, A. As cidades da América do Sul (Die Städte Südamerikas). Urbanisierung der Erde 3, vol.2, Berlin, Stuttgart: Gebr. Bornträger, 1984/85.

Teses de doutoramento sobre tópicos brasileiros, publicadas na série "Tübinger Beiträge zur Geographischen Lateinamerikaforschung" (TBGL) (“Contribuições Tübingenses para a Pesquisa sobre a América Latina")* (editor: Gerd Kohlhepp, Instituto de Geografia da Universidade de Tübingen)

Vol.2: LÜCKER, R. Processos de desenvolvimento agrário-espacial na região do Alto Uruguai. Tübingen, 1986, $278 \mathrm{p}$.

Vol.5: COY, M. Desenvolvimento regional e planejamento do desenvolvimento na periferia da Amazônia. Problemas e conflitos de interesse na colonização da frente pioneira no Estado de Rondônia. Tübingen, 1988, 549 p.

Vol.6: ACHILLES, G. Mudança na estrutura e avaliação de bairros residenciais de alto nível social no Rio de Janeiro. O desenvolvimento da metrópole brasileira sob consideração dos bairros de Ipanema e Leblon. Tübingen, 1989, 367 p.

Vol.7: GUTBERLET, J. Produção industrial e destruição do meio ambiente na região econômica de Cubatão, São Paulo. Tübingen, 1991, 338 p.

Vol.10: SOUZA, M.J. LOPES de. Pobreza, segregação sócio-espacial e conflito social na região metropolitana do Rio de Janeiro. Uma contribuição para a análise da "questão urbana" no Brasil. Tübingen, 1993, $445 \mathrm{p}$.

Vol.11: HENKEL, K. Mudança de estrutura agrária e migração no leste da Amazônia (Pará). Tübingen, 1994, $474 \mathrm{p}$.

Vol.16: LÖWEN, C.L. A relação entre desenvolvimento urbano e interdependências de centralidade da cidade de Ponta Grossa, Paraná. Uma investigação sobre o papel das cidades médias localizadas próximas às regiões metropolitanas. Tübingen, 1998, 328 p.

Vol.18: FRIEDRICH, M. Desenvolvimento urbano e problemas de planejamento de centros regionais brasileiros: Cáceres e Rondonópolis/ Mato Grosso - uma comparação. Tübingen, 1999, $312 \mathrm{p}$.

Vol.19: THEIS, I.M. Desenvolvimento e energia no sul do Brasil. Uma análise econômicogeográfica do sistema energético do Vale do Itajaí em Santa Catarina. Tübingen, 2000, 373 p. 
Vol.20: SIEDENBERG, D.R. Disparidades sócio-econômicas e política de desenvolvimento regional no Rio Grande do Sul. Uma análise sobre margem de ação, consequências e perspectivas de estratégias endógenas de desenvolvimento regional no sul do Brasil. Tübingen, 2000, $249 \mathrm{p}$.

Vol.21: BLUMENSCHEIN, M. Modificações no uso da terra na agricultura modernizada em Mato Grosso. O papel das redes, fatores institucionais e econômicos para as inovações agro-econômicas na Chapada dos Parecis. Tübingen, 2001, 376 p.

Vol.22: RÖPER, M. Criação e implementação de unidades de conservação, uma abordagem sócio-geográfica. Estudos de caso da região do Pantanal. Tübingen, 2001, 485 p.

Vol.23: NEUBURGER, M. Desenvolvimento da frente pioneira do hinterland de Cáceres, Mato Grosso. Degradação ecológica, vulnerabilidade e estratégias de sobrevivência dos pequenos colonos. Tübingen, 2002, $404 \mathrm{p}$.

Vol.24: PASCA, D. Conflitos por recursos naturais em Terras Indígenas na Amazônia Brasileira. Atores sociais em ambientes politizados. Tübingen, 2004, 374 p.

Vol.25: KARNOPP, E. Agricultores familiares entre agricultura convencional e orgânica. 0 exemplo da região do Vale do Rio Pardo/RS. Tübingen, 2006, 288 p.

Vol.30: SEGEBART, D. Monitoramento participativo como instrumento de realização de good local governance. Um exemplo de pesquisa-ação no Leste da Amazônia. Tübingen, 2007, $398 \mathrm{p}$.

Vol.31: ZIRKL, F. A importância do saneamento básico para o desenvolvimento urbano sustentável no Brasil. $O$ caso de Curitiba. Tübingen, 2007, 379 p.

*(Os títulos em idioma alemão foram traduzidos para o português). 